\title{
Nrf2 null enhances UVB-induced skin inflammation and extracellular matrix damages
}

Constance Lay Lay Saw ${ }^{1,2}$, Anne Yuqing Yang ${ }^{1,2}$, Mou-Tuan Huang ${ }^{1,3}$, Yue Liu ${ }^{1,3}$, Jong Hun Lee ${ }^{1,2}$, Tin Oo Khor ${ }^{1,2}$,

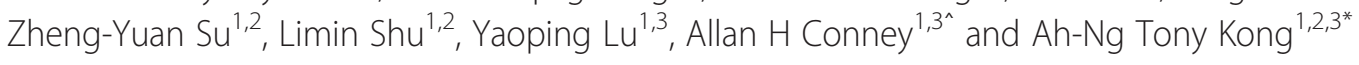

\begin{abstract}
Nrf2 plays a critical role in defending against oxidative stress and inflammation. We previously reported that Nrf2 confers protection against ultraviolet-B (UVB)-induced inflammation, sunburn reaction, and is involved in sulforaphane-mediated photo-protective effects in the skin. In this study, we aimed to demonstrate the protective role of Nrf2 against inflammation-mediated extracellular matrix (ECM) damage induced by UVB irradiation. Ear biopsy weights were significantly increased in both Nrf2 wild-type (Nrf2 WT) and knockout (Nrf2 KO) mice one week after UVB irradiation. However, these weights increased more significantly in KO mice compared to WT mice, suggesting a greater inflammatory response in $\mathrm{KO}$ mice. In addition, we analyzed the protein expression of numerous markers, including macrophage inflammatory protein-2 (MIP-2), pro-matrix metalloproteinase-9 (MMP-9), and p53. p53, a regulator of DNA repair, was overexpressed in Nrf2 KO mice, indicating that the absence of Nrf2 led to more sustained DNA damage. There was also more substantial ECM degradation and increased inflammation in UVB-irradiated Nrf2 KO mice compared to UVB-irradiated WT mice. Furthermore, the protective effects of Nrf2 in response to UVB irradiation were mediated by increased HO-1 protein expression. Collectively, our results show that Nrf2 plays a key role in protecting against UVB irradiation and that the photo-protective effect of Nrf2 is closely related to the inhibition of ECM degradation and inflammation.
\end{abstract}

Keywords: Nuclear factor (erythroid-derived 2)-like 2 (NFE2L2 or Nrf2), Ultraviolet, Inflammation, p53, pro-MMP-9, MIP-2, ECM

\section{Introduction}

The incidence of non-melanoma skin cancer has steadily increased, and ultraviolet (UV) light is one of the major causes of non-melanoma skin cancer. In fact, UV-induced nonmelanoma skin cancer is the most common type of cancer in the US and worldwide [1,2]. UVB is the most potent form of UV radiation; exposure to UVB radiation both burns the skin and drives the initiation, promotion, and progression of skin carcinogenesis [3]. UV irradiation induces the delayed expression of UV-responsive genes such as matrix metalloproteinases (MMPs), which degrade macromolecular components of the extracellular matrix (ECM), and these changes represent a hallmark of carcinogenesis and aging

\footnotetext{
* Correspondence: kongt@pharmacy.rutgers.edu

'Deceased

${ }^{1}$ Center for Cancer Prevention Research, Ernest Mario School of Pharmacy, Rutgers, The State University of New Jersey, Piscataway, NJ 08854, USA ${ }^{2}$ Department of Pharmaceutics, Ernest Mario School of Pharmacy, Rutgers, The State University of New Jersey, Piscataway, NJ 08854, USA Full list of author information is available at the end of the article
}

[4]. Moreover, altered ECM metabolism has been implicated in various diseases [5]. Oxidative stress, such as that caused by excessive reactive oxygen species (ROS), is caused by UVB damage to DNA, protein, and lipids and can lead to inflammation, gene mutation, and immunosuppression [6,7]. Due to the critical role of ROS in UVB-induced photo-carcinogenesis, ROS detoxification mechanisms have emerged as effective approaches for cancer prevention [8]. Nuclear factor erythroid-2 (NF-E2)-related factor 2 (Nrf2), a member of the cap ' $n$ ' collar family of redox-sensitive bZIP (basic leucine zipper) proteins, is the major transcriptional regulator of the expression of genes encoding phase II detoxifying/antioxidant enzymes, including heme oxygenase-1 (HO-1) [7]. HO-1 exhibits broad cytoprotective effects in various inflammatory diseases [9] and was shown to play a critical protective role in limiting oxidative damage in a UVB-induced model [10]. MMP-9 was reported to be upregulated in angiogenic dysplasias and invasive cancers of the epidermis in a mouse model of multistage 
tumorigenesis. Notably, MMP-9 is predominantly expressed in neutrophils, macrophages, and mast cells rather than oncogene-positive neoplastic cells [11]. It has also been reported that the disruption of $\mathrm{Nrf} 2$ enhanced the upregulation of key inflammatory transcription factors, such as nuclear factor kappa B (NF-kB) and MMP-9 [12], and increased expression of macrophage inflammatory protein-2 (MIP-2), a neutrophil chemotactic factor, was observed in response to 12-O-tetradecanoylphorbol-13-acetate (TPA)induced epidermal hyperplasia in mice [13]. The p53 tumor suppressor gene plays an important role in protecting cells against DNA damage and strand breaks [14], and the impact of UVB on p53 has been experimentally verified [15]. Moreover, p53 is known to be activated by DNA damage [14], oxidative stress [16], and inflammation $[17,18]$.

We previously reported the critical role of nuclear Nrf2 in the classical 2-stage model of skin carcinogenesis induced by the chemical carcinogen 7,12-dimethylbenz(a) anthracene (DMBA) and the tumor promoter TPA in Nrf2 knockout (Nrf2 KO) and wild-type (WT) mice [19]. We also recently reported that Nrf2 plays an important role in the SFN-mediated protective effects against UVBinduced inflammation [20]. To explore the potential impact of Nrf2 on UVB-induced inflammatory ECM damage in the skin, we studied the potential biomarkers involved in ECM degradation and inflammation in Nrf2 $\mathrm{KO}$ and WT mice, as these markers may be relevant to human skin carcinogenesis and photoaging [8,21-24]. Based on this context, our current study aimed to ascertain the role of Nrf2 in UVB-induced ECM damage by focusing on inflammation and DNA repair in Nrf2 $\mathrm{KO}$ and Nrf2 WT mice.

\section{Materials and methods \\ Animals}

Male and female Nrf2 KO and WT C57BL/6 mice were obtained as previously described [19]. The offspring of the eighth generation of $\mathrm{Nrf} 2 \mathrm{KO}$ mice on the C57BL/6 background were used in this study. The genotype of each animal was confirmed using DNA extracted from the tail and analyzed by PCR. Nrf2 WT mice were purchased from The Jackson Laboratory (Bar Harbor, ME, USA). Mice were housed at the Rutgers Animal Facility, maintained under 12-h light/dark cycles, and provided ad libitum access to food and water. Animal experiments were performed in accordance with the Guide for the Care and Use of Laboratory Animals. All animal experimental procedures were approved by the Animal Care and Use Committee of Rutgers, The State University of New Jersey (Protocol Number: 04-003).

\section{UV lamps}

To induce skin inflammation, animals received a single dose of UVB light $\left(300 \mathrm{~mJ} / \mathrm{cm}^{2}\right)$ as previously described
[20,25]. These UV lamps (FS72T12-UVB-HO; National Biological Corp., Twinsburg, OH, USA) emit little or no radiation $<280 \mathrm{~nm}$ and $>375 \mathrm{~nm}$. The lamps emit UVB (280-320 nm; 75-80\% of total energy) and UVA (320$375 \mathrm{~nm} ; 20-25 \%$ of total energy), as described in our previous studies $[20,25]$. The dose of UVB was quantified using a UVB Spectra 305 dosimeter (Daavlin Co., Bryan, $\mathrm{OH}, \mathrm{USA})$. The radiation was calibrated with an IL-1700 research radiometer/photometer (International Light Inc., Newburyport, MA, USA).

\section{Experimental design}

Experiments were performed in Nrf2 WT and $\mathrm{KO}$ mice to determine whether a single dose of $300 \mathrm{~mJ} / \mathrm{cm}^{2} \mathrm{UVB}$ irradiation would be sufficient to induce inflammatory reactions in the skin. Seven days after UVB irradiation, ear punches $(6 \mathrm{~mm}$ in diameter) were obtained and weighed. Two sets of experiments were conducted in Nrf2 WT and KO mice after UVB exposure (at $8 \mathrm{~h}$ and 8 days). For each experiment, 4 groups of mice were evaluated: (1) no UVB in Nrf2 WT mice; (2) UVB in Nrf2 WT mice; (3) no UVB in Nrf2 KO mice; and (4) UVB in Nrf2 KO mice. A minimum of three animals was included in each group. The hair on the dorsal region of each mouse was removed 2 days before UVB irradiation, as previously described [20]. At the end of the experiment, the mice were sacrificed by cervical dislocation, and the skin samples were frozen in liquid nitrogen and stored at $-80^{\circ} \mathrm{C}$.

\section{Preparation of skin specimens and histological examination}

Skin samples were obtained from the dorsal area of the mouse and were placed in 10\% phosphate-buffered formalin at room temperature overnight. The samples were then dehydrated in increasing concentrations (80, 95, and $100 \%)$ of ethanol, cleared in xylene, and embedded in Paraplast Plus (Fisher Scientific, Pittsburgh, PA, USA). Four-micrometer serial sections were cut from the skin block, deparaffinized, rehydrated, and stained with hematoxylin and eosin $(\mathrm{H} \& \mathrm{E})$. The $\mathrm{H} \& \mathrm{E}$ sections were examined under a light microscope (Nikon Eclipse E600, Japan).

\section{ELISA for pro-inflammatory proteins and p53}

The protein levels of pro-inflammatory cytokines and p53 were determined using two-site sandwich enzymelinked immunosorbent assays (ELISAs). Dorsal skin tissues were homogenized in phosphate-buffered saline (PBS) containing $0.4 \mathrm{M} \mathrm{NaCl}, 0.05 \%$ Tween-20, $0.5 \%$ bovine serum albumin, $0.1 \mathrm{mM}$ phenylmethylsulphonyl fluoride, $0.1 \mathrm{mM}$ benzethonium chloride, $10 \mathrm{mM}$ EDTA, and 200 KIU aprotinin per $\mathrm{mL}$. The homogenates were centrifuged at $12,000 \times g$ for $15 \mathrm{~min}$ at $4^{\circ} \mathrm{C}$. The supernatant was used for the determination of cytokines levels. Pro-matrix 
metalloproteinase-9 (pro-MMP-9; DY909), macrophage inflammatory protein-2 (MIP-2; DY452), and p53 (DYC1746-2) kits were purchased from R\&D Systems, Inc. (Minneapolis, MN, USA).

\section{Western blotting}

The protein samples were prepared as described above for the ELISAs. The protein concentration was measured using Bio-Rad protein assay reagent (Hercules, CA, USA). Equal amounts of protein $(50 \mu \mathrm{g})$ from each sample were loaded onto a homemade $10 \%$ Tris/glycine SDSpolyacrylamide gel containing $0.1 \%$ SDS and run at $200 \mathrm{~V}$ for $1 \mathrm{~h}$. The proteins were transferred to a nitrocellulose membrane by electroblotting using a Bio-Rad semidry transfer blotting apparatus over $1.5 \mathrm{~h}$ at $90 \mathrm{~V}$ (Bio-Rad, Hercules, CA, USA). The membrane was blocked with blocking buffer (Odyssey system, LI-COR Biosciences, Lincoln, NE, USA) for $1 \mathrm{~h}$ at room temperature. The primary antibodies were diluted in the same blocking buffer and were added to detect the target proteins. After an overnight incubation at $4{ }^{\circ} \mathrm{C}$, the membrane was washed with TBST (Tris-buffered saline and $0.05 \%$ Tween-20) and then incubated with the secondary antibody. Finally, the target protein bands were visualized with an Odyssey infrared imager. Antibodies against $\beta$-actin and HO-1 were purchased from Santa Cruz Biotechnology, Inc. (CA, USA). Densitometry was performed using the Image J software (version 1.44, National Institute of Health, USA). The relative protein expression was obtained by first calculating the relative intensity compared to the control (no UVB treatment) sample on the same blot and then normalizing this value to the intensity of $\beta$-actin from the same sample.

\section{Data presentation and statistical analysis}

The data are presented as the mean \pm standard error of the mean (SEM), except as otherwise stated. Student's $t$-test was used to determine statistically significant differences between groups. A p value $<0.05$ was considered statistically significant.

\section{Results}

A single dose of $300 \mathrm{~mJ} / \mathrm{cm}^{2}$ UVB significantly increased the ear biopsy weight, and Nrf2 KO mice were more susceptible to UVB-induced skin edema

To determine the effect of Nrf2 on UVB-induced trauma to mouse skin, we irritated mouse skin with a single dose of UVB $\left(300 \mathrm{~mJ} / \mathrm{cm}^{2}\right)$ and measured the ear biopsy weight 1 week after exposure. In the untreated group, the average ear weights of the Nrf2 WT and KO mice were $7.28 \pm 0.24 \mathrm{mg}$ and $6.57 \pm 0.42 \mathrm{mg}$, respectively. In the UVB-treated group, these weights increased to $8.45 \pm$ $0.42 \mathrm{mg}$ in Nrf2 WT mice and to $13.13 \pm 0.45 \mathrm{mg}$ in Nrf2 KO mice (Figure 1A). UVB exposure significantly
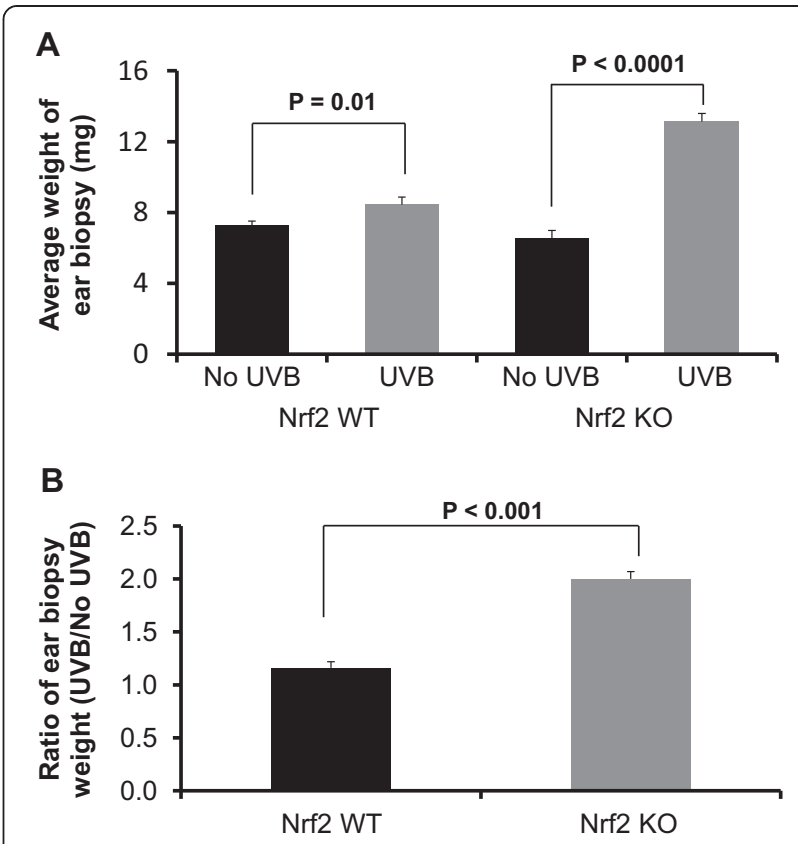

Figure 1 Ear biopsy weights in Nrf2 WT and KO mice after UVB exposure. (A) Nrf2WT and KO mice were treated with or without a single dose of UVB $\left(300 \mathrm{~mJ} / \mathrm{cm}^{2}\right)$. The mice were sacrificed 1 week after UVB irradiation. Ear punches (6 $\mathrm{mm}$ in diameter) were taken and weighed. (B) The ratio of the ear punch weight in UVB-treated versus control skin suggested that Nrf2 significantly protected against the UVB-induced increase in ear punch weight $(p<0.05)$. The data are presented as the mean \pm SEM.

increased the ear biopsy weight in $\mathrm{Nrf} 2 \mathrm{KO}$ mice by nearly $100 \%$, whereas there was only a $16 \%$ increase observed in Nrf2 WT mice. The ear biopsy weight ratio indicated that Nrf2 $\mathrm{KO}$ mice were more susceptible to UVB-induced skin edema (Figure 1B). These data demonstrated that a single dose of UVB $\left(300 \mathrm{~mJ} / \mathrm{cm}^{2}\right)$ was sufficient to cause UVB-induced inflammatory damage to mouse skin, especially in Nrf2 $\mathrm{KO}$ mice. This single dose of UVB was used in all the following experiments.

\section{The absence of Nrf2 gene expression increased the UVB-induced skin thickness}

To determine the effect of Nrf2 in mouse skin exposed to UVB irritation, we treated the dorsal skin area with a single dose of UVB $\left(300 \mathrm{~mJ} / \mathrm{cm}^{2}\right)$ and took a skin biopsy 8 days later. Nrf2 $\mathrm{KO}$ mice exhibited a significant increase in skin thickness 8 days after UVB irradiation (Figure 2), and a single dose of UVB $\left(300 \mathrm{~mJ} / \mathrm{cm}^{2}\right)$ led to a greater increase in skin thickness in $\mathrm{Nrf} 2 \mathrm{KO}$ mice compared to Nrf2 WT mice (Figure 2).

\section{Nrf2 KO mice were significantly more susceptible to} UVB-induced inflammation and ECM degradation

To characterize the UVB-induced inflammatory damage to the ECM, we measured the expression of biomarkers 

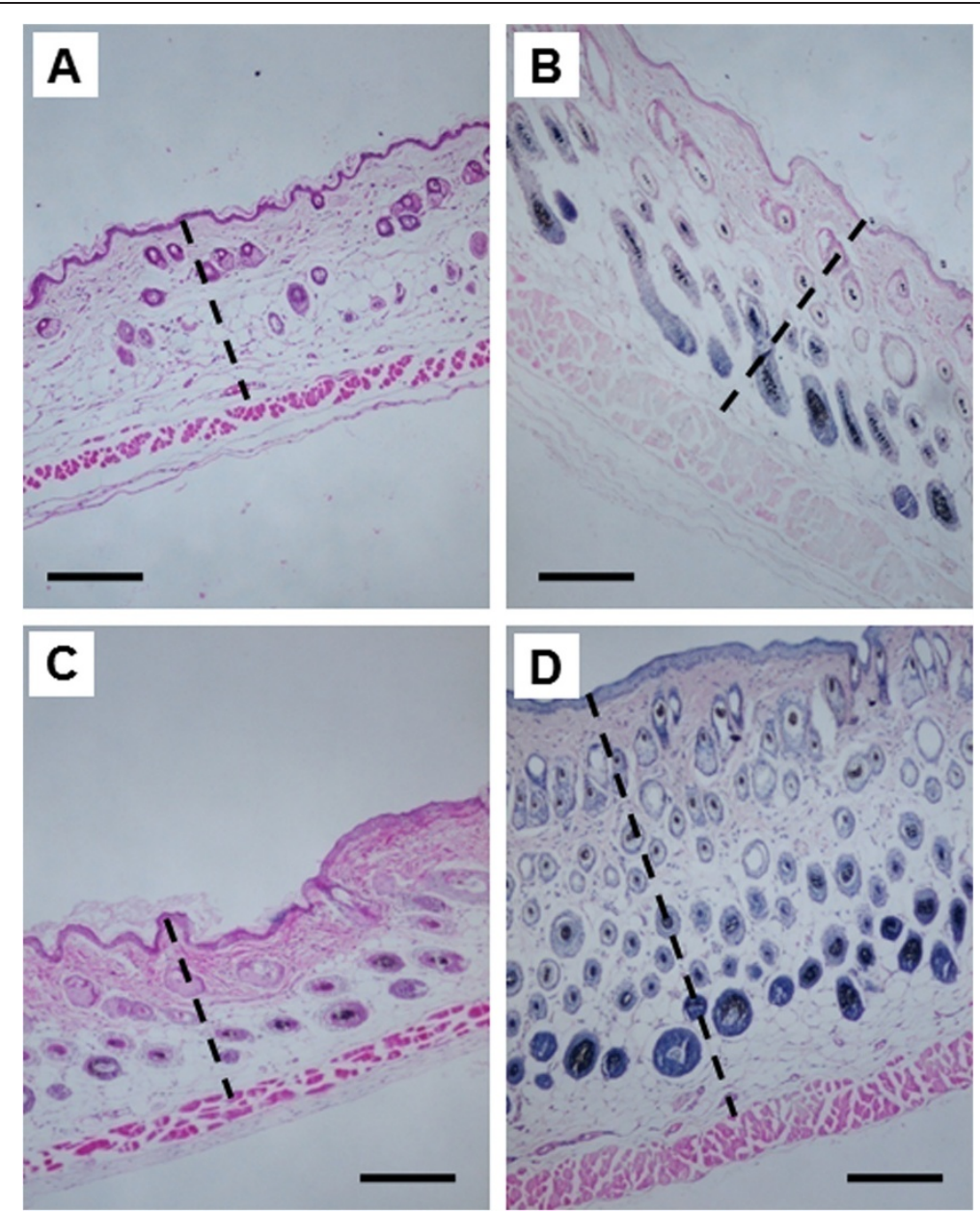

Figure 2 Skin thickness in Nrf2 WT versus Nrf2 KO mice after UVB exposure. H\&E staining was performed in skin samples 8 days after treatment with a single dose of UVB $\left(300 \mathrm{~mJ} / \mathrm{cm}^{2}\right)$. WT mice were treated (A) without UVB or (B) with UVB. KO mice were treated (C) without UVB or (D) with UVB. The horizontal line represents $200 \mu \mathrm{m}$, and the broken lines indicate the skin thickness. Original magnification, $\times 400$. The Nrf2 KO mice exhibited more edema and inflammatory changes, such as increased skin thickness, whereas the Nrf2 WT mice demonstrated fewer changes; these data correlated with the ear punch weights (Figure 1) and other biomarkers (Figures 3, 4, 5).

related to inflammation (MIP-2) and ECM degradation (pro-MMP-9) $8 \mathrm{~h}$ after UVB exposure. A single dose of UVB $\left(300 \mathrm{~mJ} / \mathrm{cm}^{2}\right)$ significantly upregulated the protein expression of MIP-2 and pro-MMP-9 in both Nrf2 WT and $\mathrm{KO}$ mice (Figures $3 \mathrm{~A}$ and $3 \mathrm{C}$ ). However, after UVB irradiation, these biomarkers were more significantly upregulated in Nrf2 $\mathrm{KO}$ mice compared to WT mice (Figures 3B and 3D).

\section{Nrf2 KO mice were more susceptible to UVB-induced upregulation of $\mathrm{p} 53$}

To evaluate sustained DNA damage, we examined p53 protein expression $8 \mathrm{~h}$ after UVB irradiation. In both Nrf2 WT and KO mice, a single dose of UVB $\left(300 \mathrm{~mJ} / \mathrm{cm}^{2}\right)$ significantly increased $\mathrm{p} 53$ protein expression compared to untreated skin (Figure 4). However, there was a greater increase in p53 expression in Nrf2 $\mathrm{KO}$ mice compared to Nrf2 WT mice.

\section{UVB increased expression of the Nrf2 target HO-1, an} antioxidant biomarker, in Nrf2 WT mice compared to Nrf2 KO mice

To elucidate the mechanism responsible for the photoprotective effect of Nrf2 in UVB-exposed mice, Nrf2 WT and KO mice were exposed to UVB irradiation and sacrificed $8 \mathrm{~h}$ later. We measured the expression of $\mathrm{HO}-1$, an anti-oxidative enzyme downstream of Nrf2, in Nrf2 WT and KO mice and compared these levels to those observed in untreated control Nrf2 WT and KO mice. Compared to untreated WT mice, HO-1 protein expression was significantly increased in UVB-treated Nrf2 WT mice $(\mathrm{p}<0.05)$, whereas HO-1 expression was not significantly altered in Nrf2 KO mice (Figure 5). 

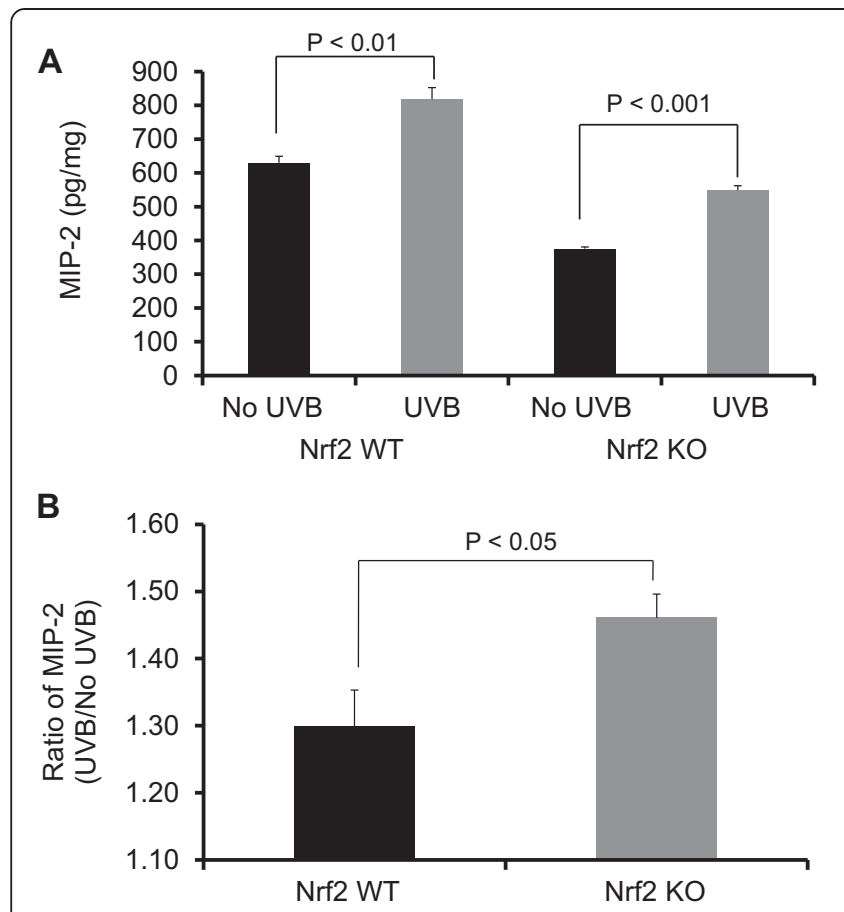
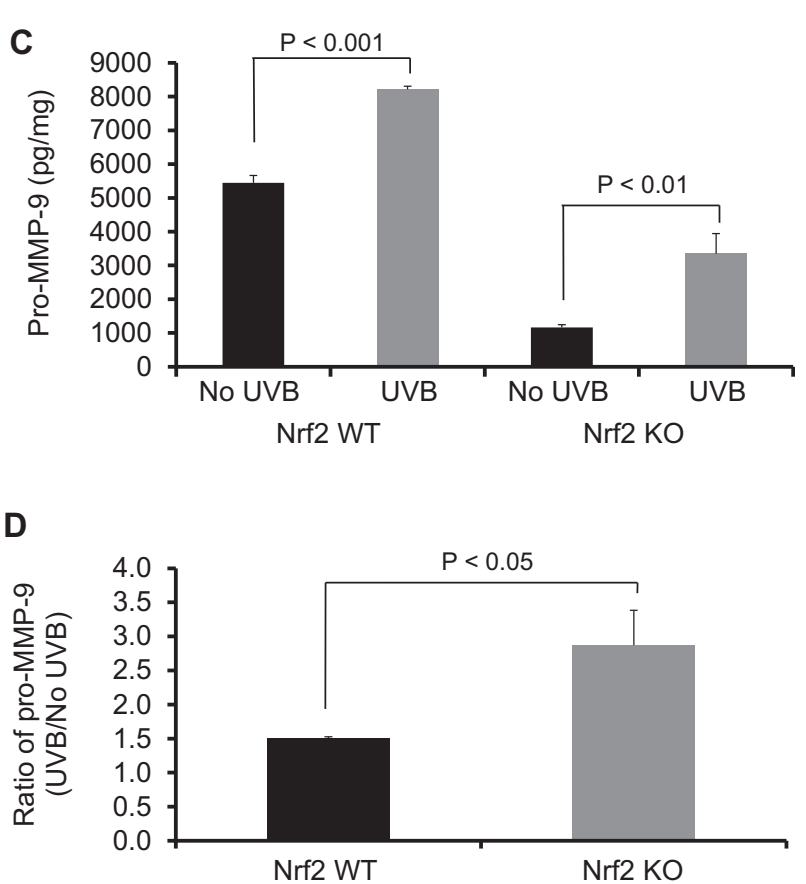

Figure 3 MIP-2 and Pro-MMP-9 expression in Nrf2 WT versus Nrf2 KO mice after UVB exposure. Nrf2 WT and KO mice were irradiated with a single dose of UVB $\left(300 \mathrm{~mJ} / \mathrm{cm}^{2}\right)$. After $8 \mathrm{~h},(\mathbf{A})$ the MIP-2 expression and (B) the ratios of MIP-2 protein levels in WT and Nrf2 KO mice; (C) the Pro-MMP-9 expression and (D) the ratios of Pro-MMP-9 protein levels in UVB-treated versus control skin (No UVB-treated) were determined; Nrf2 significantly protected against UVB-induced the MIP-2 and pro-MMP-9 expression $(p<0.05)$. The data are presented as the mean \pm SEM.

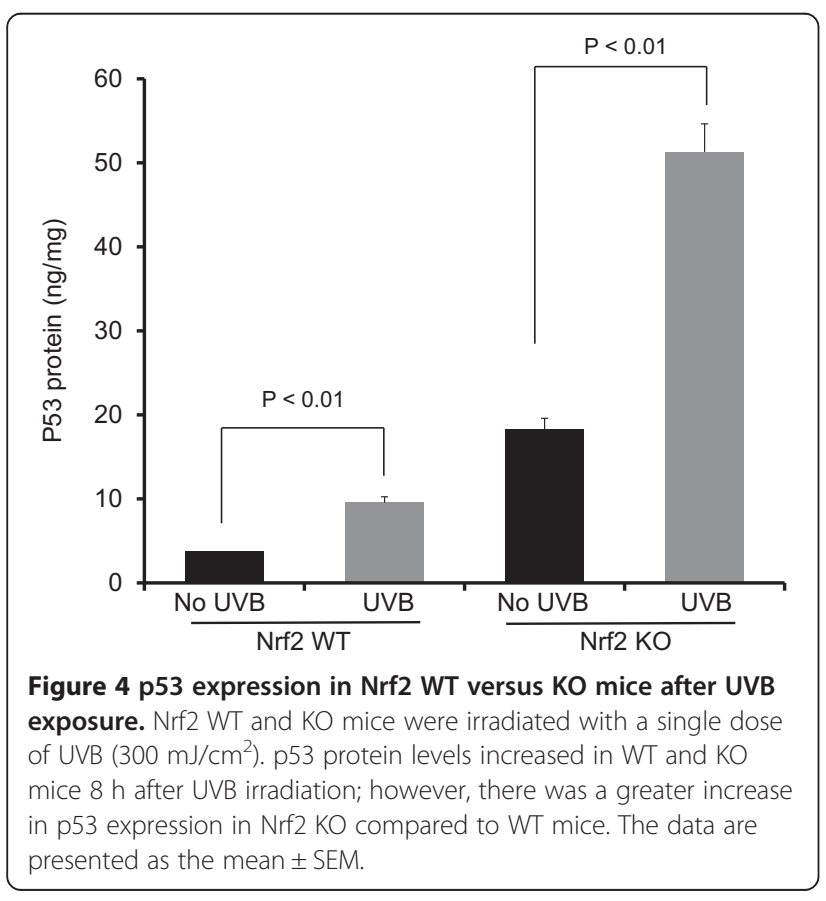

In particular, UVB exposure increased $\mathrm{HO}-1$ expression by $43 \%$ in Nrf2 WT mice, which was much higher than the $24 \%$ increase observed in Nrf2 KO mice.

\section{Discussion}

In the present study, we investigated the photo-protective role of Nrf2 in inflammation-mediated ECM damage induced by UV, and our results indicated that Nrf2 KO mice were more susceptible to UVB radiation. Erythema has long been used as an indicator of UV-induced inflammation [26] and has been characterized as a reliable, reproducible and quantitative measurement of the degree of UV-induced inflammation [27]. Thus, measuring the weight or thickness of irritated skin is a reliable indicator of inflammation $[20,28]$. The results presented here demonstrated that UVB exposure significantly increased the ear biopsy weight in Nrf2 KO mice by almost $100 \%$, whereas the ear biopsy weight only increased by $16 \%$ in Nrf2 WT mice. Moreover, the ratio of ear biopsy weights suggested that Nrf2 KO mice were more susceptible to UVB-induced skin edema (Figure 1). In addition, Nrf2 KO mice exhibited a greater increase in skin thickness after UVB exposure (Figure 2), which suggested that the loss of Nrf2 resulted in increased sensitivity to UVB radiation, thereby implying that there was more active inflammation in Nrf2-deficient mice. Therefore, we performed various biological assays to demonstrate that the observed 


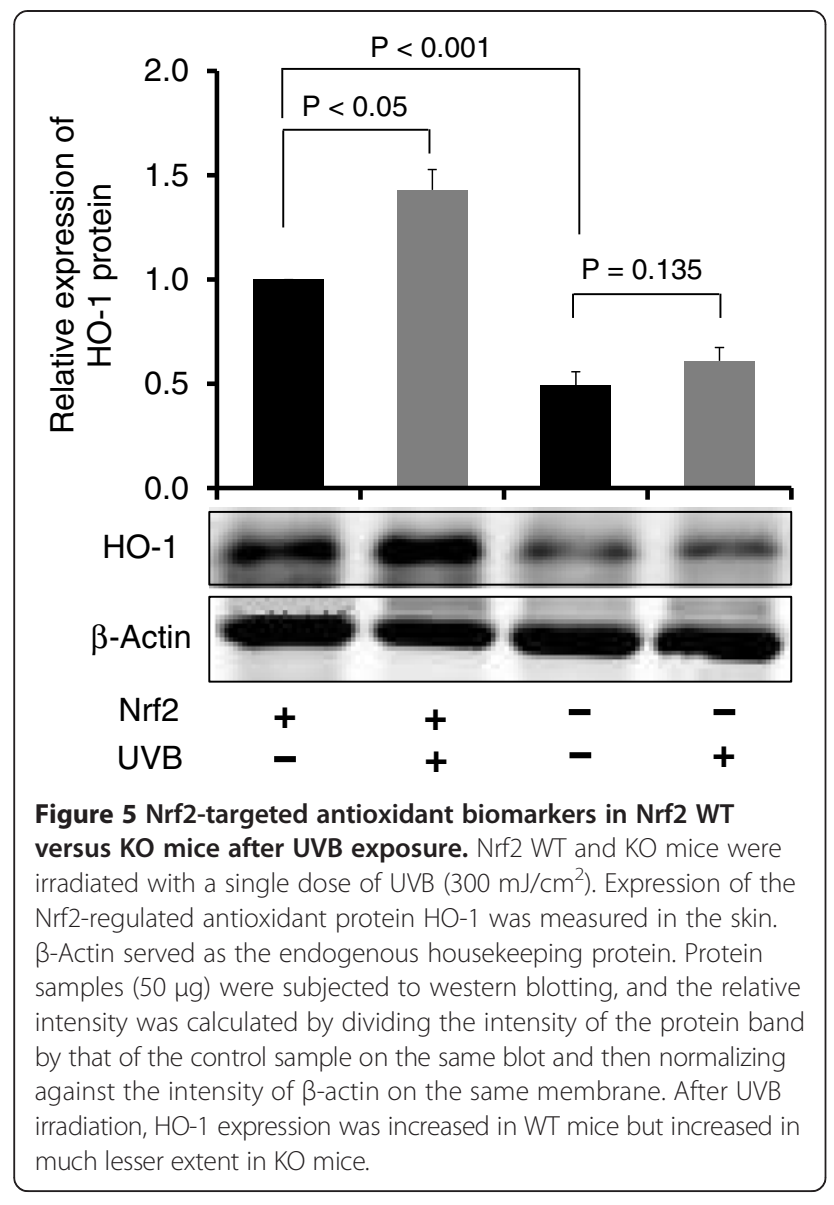

UVB-induced responses were related to inflammation, ECM stability, and DNA damage. It has been reported in in vivo studies of human skin that UV irradiation significantly affects the coordinated regulation of various MMPs; after a single exposure to UV, MMP-9 activity increased 4-fold compared to non-UV irradiated human skin in a time-dependent manner [29]. MIP-2, a key mediator of neutrophil recruitment, is involved in the early infiltration of neutrophils into UVB-exposed skin [30]. In our study, UVB irradiation significantly enhanced the expression of MIP-2 in both Nrf2 WT and KO mice (Figure 3A). However, the increase in MIP-2 expression was much greater in Nrf2 $\mathrm{KO}$ mice compared to WT mice (Figure 3B). These MIP-2 findings correlate with those of a previous study in which MIP-2 expression was increased in mice after UVB irradiation [30]. Photoaged skin displays prominent alterations in the collagenous ECM of connective tissue [29], and the detrimental effects of ECM degradation include diminished structural integrity, impaired wound healing, loss of cell viability, and cancer metastasis [31]. There is growing interest in understanding the role of aberrant ECM modifications, especially regarding the "cancer stem cell niche" and the "metastatic niche" during key stages of cancer progression [7,32]. Pro-MMP-9 expression was increased significantly in Nrf2 $\mathrm{KO}$ mice compared to WT mice (Figures $3 \mathrm{C}$ and $3 \mathrm{D}$ ), and these results indicated that the absence of Nrf2 augmented the expression of matrix-degrading proteinases, including pro-MMP-9, in response to UVB irradiation. Furthermore, many ECM fragments that are created via degradation by various MMPs act as chemotactic factors that cause endothelial and inflammatory cells to migrate into areas of active tumor cell proliferation and growth [33]. Thus, the photo-protective effect of Nrf2 was mediated by a reduction in MMP-9 expression. The p53 tumor suppressor gene is typically activated by DNA damage [14], oxidative stress [16], and/or inflammation $[17,18]$, and we observed that both of Nrf2 WT and KO mice exhibited increased p53 expression in response to UVB exposure, although the p53 expression was higher in KO mice. Regarding the role of p53 in DNA repair, increased p53 expression correlates with increased DNA damage, suggesting that the loss of Nrf2 may fail to protect DNA but could enable abundant p53 to repair the DNA damage. Indeed, a previous study showed that the extent of DNA damage correlates with the amount of UV exposure in various skin cells and models [34]. In our study, the higher p53 expression observed in Nrf2 KO mice after UVB exposure could be an indicator of more sustained DNA damage in need of repair. HO- 1 is a downstream antioxidant enzyme that is regulated by Nrf2 and has a broad cytoprotective effect in various inflammatory diseases and UVB irradiation $[9,10]$. In our study, UVB exposure enhanced HO-1 expression by $43 \%$ in Nrf2 WT mice and by $24 \%$ in Nrf2 $\mathrm{KO}$ mice (Figure 5), which indicated that the photoprotective effect of Nrf2 was mediated by $\mathrm{HO}-1$.

\section{Conclusions}

UVB irradiation induces aberrant biological responses, such as increased skin erythema and thickness, up-regulated expression of inflammatory and ECM-degrading enzymes, and augmented DNA damage. According to our results, these biological responses were milder in WT mice compared to Nrf2 KO mice. In addition, our UVB-induced mechanistic study revealed that the protective effect of Nrf2 was mediated by HO-1, and these findings suggest that Nrf2 plays a key role in protecting against UVB irradiation. Moreover, our study is the first to demonstrate that the photo-protective effect of Nrf2 is closely related to the inhibition of ECM degradation and inflammation through the overexpression of HO- 1 .

\section{Competing interests}

The authors declare that they have no competing interests. 


\section{Authors' contributions}

CLLS, MTH and YL conceived the research design and carried out the experiments, as well as analyzed the data. CLLS, AYY, JHL, TOK, ZYS, LS and ANTK wrote, reviewed and/or revised this manuscript. MTH, YPL, AHC and ANTK provided administrative, technical and material support. All authors read and approved the final manuscript.

\section{Acknowledgments}

The authors would like to thank the members of Tony Kong's laboratory for helpful discussions. This work was supported in part by institutional funds.

\section{Author details}

${ }^{1}$ Center for Cancer Prevention Research, Ernest Mario School of Pharmacy, Rutgers, The State University of New Jersey, Piscataway, NJ 08854, USA. ${ }^{2}$ Department of Pharmaceutics, Ernest Mario School of Pharmacy, Rutgers, The State University of New Jersey, Piscataway, NJ 08854, USA. ${ }^{3}$ Susan Lehman Cullman Laboratory for Cancer Research, Department of Chemical Biology, Ernest Mario School of Pharmacy, Rutgers, The State University of New Jersey, Piscataway, NJ 08854, USA.

Received: 13 March 2014 Accepted: 17 June 2014

Published: 5 August 2014

\section{References}

1. Stern RS: Prevalence of a history of skin cancer in 2007: results of an incidence-based model. Arch Dermatol 2010, 146(3):279-282.

2. Diepgen TL, Mahler V: The epidemiology of skin cancer. Br J Dermatol 2002, 146(Suppl 61):1-6.

3. Brash DE, Ziegler A, Jonason AS, Simon JA, Kunala S, Leffell DJ: Sunlight and sunburn in human skin cancer: p53, apoptosis, and tumor promotion. J Investig Dermatol Symp Proc 1996, 1(2):136-142.

4. Brenneisen P. Sies H, Scharffetter-Kochanek K: Ultraviolet-B irradiation and matrix metalloproteinases - From induction via signaling to initial events, in Cell Signaling, Transcription, and Translation as Therapeutic Targets. Edited by Diederich M. New York: Acad Sciences; 2002:31-43.

5. Hansson J, Vasan RS, Ärnlöv J, Ingelsson E, Lind L, Larsson A, Michaëlsson K, Sundstrom J: Biomarkers of extracellular matrix metabolism (MMP-9 and TIMP-1) and risk of stroke, myocardial infarction, and cause-specific mortality: cohort study. PLoS One 2011, 6(1):e16185.

6. Afaq F, Adhami VM, Mukhtar H: Photochemoprevention of ultraviolet B signaling and photocarcinogenesis. Mutat Res Fund Mol Mech Mutagen 2005, 571(1-2):153-173.

7. Lee JH, Khor TO, Shu L, Su ZY, Fuentes F, Kong AN: Dietary phytochemicals and cancer prevention: Nrf2 signaling, epigenetics, and cell death mechanisms in blocking cancer initiation and progression. Pharmacol Ther 2012, 137(2):153-171

8. Schafer M, Dutsch S, auf dem Keller U, Navid F, Schwarz A, Johnson DA Johnson JA, Werner S: Nrf2 establishes a glutathione-mediated gradient of UVB cytoprotection in the epidermis. Genes Dev 2010, 24(10):1045-1058.

9. Gozzelino R, Jeney V, Soares MP: Mechanisms of Cell Protection by Heme Oxygenase-1, Annual Review of Pharmacology and Toxicology. Palo Alto: Annual Reviews; 2010:323-354.

10. Black AT, Gordon MK, Heck DE, Gallo MA, Laskin DL, Laskin JD: UVB light regulates expression of antioxidants and inflammatory mediators in human corneal epithelial cells. Biochem Pharmacol 2011, 81(7):873-880.

11. Coussens LM, Tinkle CL, Hanahan D, Werb Z: MMP-9 supplied by bone marrow-derived cells contributes to skin carcinogenesis. Cell 2000, 103(3):481-490.

12. Mao $L$, Wang $H$, Qiao $L$, Wang $X$ : Disruption of Nrf2 enhances the upregulation of nuclear factor-kappaB activity, tumor necrosis factor-alpha, and matrix metalloproteinase- 9 after spinal cord injury in mice. Mediat Inflamm 2010, 2010:238321.

13. Wang HQ, Smart RC: Overexpression of protein kinase C-alpha in the epidermis of transgenic mice results in striking alterations in phorbol ester-induced inflammation and COX-2, MIP-2 and TNF-alpha expression but not tumor promotion. J Cell Sci 1999, 112(20):3497-3506.

14. Nelson WG, Kastan MB: DNA strand breaks: the DNA template alterations that trigger p53-dependent DNA damage response pathways. Mol Cell Biol 1994, 14(3):1815-1823.
15. van Kranen HJ, de Laat A, van de Ven J, Wester PW, de Vries A, Berg RJ, van Kreijl CF, de Gruijl FR: Low incidence of p53 mutations in UVA (365-nm)induced skin tumors in hairless mice. Canc Res 1997, 57(7):1238-1240.

16. Han ES, Muller FL, Perez VI, Qi W, Liang H, Xi L, Fu C, Doyle E, Hickey M, Cornell J, Epstein CJ, Roberts $L$, Van Remmen H, Richardson A: The in vivo gene expression signature of oxidative stress. Physiol Genomics 2008, 34(1):112-126

17. Ak P, Levine AJ: $\mathbf{p} 53$ and NF-kappaB: different strategies for responding to stress lead to a functional antagonism. Faseb J 2010, 24(10):3643-3652.

18. Reuter S, Gupta SC, Chaturvedi MM, Aggarwal BB: Oxidative stress, inflammation, and cancer: how are they linked? Free Radic Biol Med 2010 49(11):1603-1616.

19. Xu C, Huang MT, Shen G, Yuan X, Lin W, Khor TO, Conney AH, Kong AN: Inhibition of 7,12-dimethylbenz(a)anthracene-induced skin tumorigenesis in C57BL/6 mice by sulforaphane is mediated by nuclear factor E2-related factor 2. Canc Res 2006, 66(16):8293-8296.

20. Saw CL, Huang MT, Liu Y, Khor TO, Conney AH, Kong AN: Impact of Nrf2 on UVB-induced skin inflammation/photoprotection and photoprotective effect of sulforaphane. Mol Carcinog 2011, 50(6):479-486.

21. Marrot L, Jones C, Perez P, Meunier JR: The significance of Nrf2 pathway in (photo)-oxidative stress response in melanocytes and keratinocytes of the human epidermis. Pigment Cell Melanoma Res 2008, 21(1):79-88.

22. auf dem Keller $U$, Huber M, Beyer TA, Kumin A, Siemes C, Braun S, Bugnon P. Mitropoulos V, Johnson DA, Johnson JA, Hohl D, Werner S: Nrf transcription factors in keratinocytes are essential for skin tumor prevention but not for wound healing. Mol Cell Biol 2006, 26(10):3773-3784.

23. Wondrak GT, Cabello CM, Villeneuve NF, Zhang S, Ley S, Li Y, Sun Z, Zhang DD: Cinnamoyl-based Nrf2-activators targeting human skin cell photo-oxidative stress. Free Radic Biol Med 2008, 45(4):385-395.

24. MacLeod AK, McMahon M, Plummer SM, Higgins LG, Penning TM, Igarashi K, Hayes JD: Characterization of the cancer chemopreventive NRF2-dependent gene battery in human keratinocytes: demonstration that the KEAP1-NRF2 pathway, and not the BACH1-NRF2 pathway, controls cytoprotection against electrophiles as well as redox-cycling compounds. Carcinogenesis 2009, 30(9):1571-1580

25. Lu YP, Lou YR, Yen P, Mitchell D, Huang MT, Conney AH: Time course for early adaptive responses to ultraviolet $\mathrm{B}$ light in the epidermis of SKH-1 mice. Canc Res 1999, 59(18):4591-4602.

26. Eaglstein WH, Sakai M, Mizuno N: Ultraviolet radiation-induced inflammation and leukocytes. J Invest Dermatol 1979, 72(2):59-63.

27. Cole C, Sambuco C, Forbes P, Davies R: Response to ultraviolet radiation: ear swelling in hairless mice. Photodermatol 1984, 1(3):114-118

28. Huang MT: Inhibitory effects of black tea theaflavin derivatives on 12-0-tetradecanoylphorbol-13-acetate-induced inflammation and arachidonic acid metabolism in mouse ears. Mol Nutr Food Res 2006, 50(2):115-122.

29. Fisher GJ, Wang ZQ, Datta SC, Varani J, Kang S, Voorhees JJ: Pathophysiology of premature skin aging induced by ultraviolet light. N Engl J Med 1997, 337(20):1419-1428.

30. Komatsu J, Koyama H, Maeda N, Aratani Y: Earlier onset of neutrophil-mediated inflammation in the ultraviolet-exposed skin of mice deficient in myeloperoxidase and NADPH oxidase. Inflamm Res 2006, 55(5):200-206.

31. Gonzalez S, Moran M, Kochevar IE: Chronic photodamage in skin of mast cell-deficient mice. Photochem Photobiol 1999, 70(2):248-253.

32. Lu P, Takai K, Weaver VM, Werb Z: Extracellular matrix degradation and remodeling in development and disease. Cold Spring Harb Perspect Biol 2011, 3(12).

33. Mott JD, Werb Z: Regulation of matrix biology by matrix metalloproteinases. Curr Opin Cell Biol 2004, 16(5):558-564

34. Marrot L, Planel E, Ginestet AC, Belaidi JP, Jones C, Meunier JR: In vitro tools for photobiological testing: molecular responses to simulated solar UV of keratinocytes growing as monolayers or as part of reconstructed skin. Photochem Photobiol Sci 2010, 9(4):448-458.

doi:10.1186/2045-3701-4-39

Cite this article as: Saw et al:: Nrf2 null enhances UVB-induced skin inflammation and extracellular matrix damages. Cell \& Bioscience 2014 4:39. 